\title{
Ultrasonic Measurements of Temperature in Aqueous Solutions: Why and How
}

\author{
A. Afaneh, S. Alzebda, V. Ivchenko, and A. N. Kalashnikov \\ Department of Electrical and Electronic Engineering, The University of Nottingham, University Park, Nottingham, NG7 2RD, UK \\ Correspondence should be addressed to A. N. Kalashnikov, alexander.kalashnikov@nottingham.ac.uk
}

Received 30 September 2010; Revised 12 January 2011; Accepted 14 February 2011

Academic Editor: Faramarz Farahi

Copyright () 2011 A. Afaneh et al. This is an open access article distributed under the Creative Commons Attribution License, which permits unrestricted use, distribution, and reproduction in any medium, provided the original work is properly cited.

The paper describes two different approaches to ultrasonic measurements of temperature in aqueous solutions. The first approach uses two narrowband ultrasonic transducers and support electronics that form an oscillating sensor which output frequency is related to the measured temperature. This low-cost sensor demonstrated sensitivity of about $40 \mathrm{~Hz} / \mathrm{K}$ at the distance of $190 \mathrm{~mm}$ and the operating frequency of about $25 \mathrm{kHz}$. The second approach utilised pulse-echo mode at the centre frequency of $20 \mathrm{MHz}$. The reflector featured a cavity that was filled with deionised water. The ultrasound propagation delay in the cavity was related to the temperature in the solution. The experiments were conducted for deionised water, and solutions of sodium persulfate, sodium chloride, and acetic acid with concentrations up to $0.5 \mathrm{M}$. In the experiments (conducted within the temperature range from 15 to $30^{\circ} \mathrm{C}$ ), we observed increases in the ultrasound velocity for increased temperatures and concentrations as was expected. Measurement results were compared with literature data for pure and seawater. It was concluded that ultrasonic measurements of temperature were conducted with the resolution well below $0.1 \mathrm{~K}$ for both methods. Advantages of ultrasonic temperature measurements over conventional thermometers were discussed.

\section{Introduction to Ultrasonic Evaluation of Temperature}

Ultrasonic evaluation is used for various objects and media, especially when they are opaque and thus impenetrable by electromagnetic radiation. It involves excitation of ultrasonic waves by some transducers and reception of these waves after they have passed through the whole or a part of the object under evaluation. The measured decrease in the wave's amplitude determines the ultrasound attenuation whilst the measured propagation delay specifies the ultrasound velocity. These parameters differ for various materials and also depend on the environmental conditions such as temperature (e.g., [1]). Ultrasonic spectroscopy is concerned with the ultrasound attenuation and velocity across a range of frequencies.

Ultrasonic evaluation normally employs low intensity ultrasound, and can be considered non-invasive in most cases. However, the emitted energy needs to be constrained if it can cause changes to the object under evaluation.
Ultrasonic evaluation is realised by using various arrangements for the wave excitation and reception (Figure 1) that have their specific advantages and limitations (Table 1).

Some of them require two transducers (through transmission and pitch-catch, $\mathrm{T}+\mathrm{R}$ in Table 1), while some others utilise the same transducer for transmission first and then for reception (pulse-echo and backscatter, T/R in Table 1). Acoustic emission signals are usually produced by the mechanically loaded test object itself, thus only a receiving transducer is required.

The temperature dependence of ultrasound velocity was presumably first reported back in 1873 [2]. Ultrasonic thermometers were used in the nuclear energy industry for measuring very high temperatures by placing a waveguide into a zone of interest and remotely interrogating it with ultrasonic waves [3]. They were also found useful for the measurement of the temperature of hot exhaust gases in power plants [4]. The reported temperature range for these devices was up to $20,000 \mathrm{~K}$ for gases and $2,500 \mathrm{~K}$ for waveguides with the number of instruments sold by 1975 estimated at 500 [5]. 


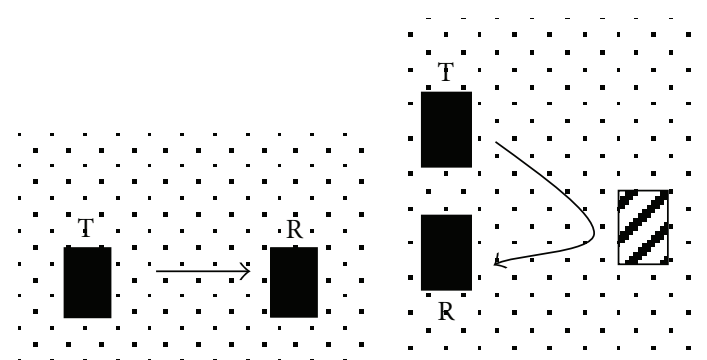

(a) (b)

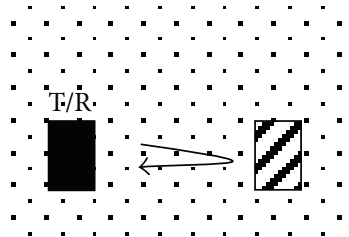

(c)

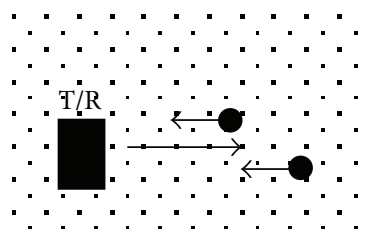

(d)

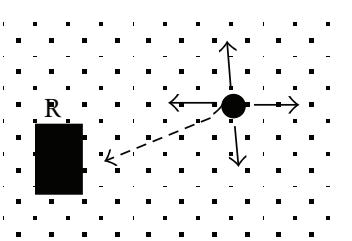

(e)

FIGURE 1: Experimental arrangements for various ultrasonic evaluation methods.

TABLE 1: Features of various ultrasonic evaluation methods.

\begin{tabular}{|c|c|c|c|c|}
\hline Method & Key to Figure 1 & Transducer & Positives & Comments \\
\hline Through transmission & (a) & $\mathrm{T}+\mathrm{R}$ & $\begin{array}{l}\text { Simpler electronics compared to } \\
\text { (c) }\end{array}$ & $\begin{array}{l}\text { Continuous wave and pulse operation are } \\
\text { possible }\end{array}$ \\
\hline Pitch-catch & (b) & $\mathrm{T}+\mathrm{R}$ & $\begin{array}{l}\text { Single side access required only } \\
\text { compared to (a) }\end{array}$ & Same as (a) \\
\hline Pulse-echo & (c) & $\mathrm{T} / \mathrm{R}$ & $\begin{array}{l}\text { Single transducer required only } \\
\text { compared to }(a, b)\end{array}$ & Pulse operation only \\
\hline Backscatter & (d) & $\mathrm{T} / \mathrm{R}$ & $\begin{array}{l}\text { No reflector required compared } \\
\text { to }(\mathrm{c})\end{array}$ & $\begin{array}{l}\text { Same as (c); effective for highly scattered } \\
\text { media }\end{array}$ \\
\hline Acoustic emission & (e) & $\mathrm{R}$ & $\begin{array}{l}\text { No need for transmitter and its } \\
\text { driver }\end{array}$ & $\begin{array}{l}\text { Signals are difficult to interpret; limited } \\
\text { applicability }\end{array}$ \\
\hline
\end{tabular}

The number of the ultrasonic thermometers being used to date remains limited because of the advantages of conventional temperature sensors (thermocouples [6], resistance temperature detectors [7], thermistors [8] and integrated silicon temperature sensors, e.g., [9]), most importantly, because of their cost. The cost of a single conventional temperature sensor is usually below $\$ 10$, and the cost of a complete instrument with a resolution of $0.1 \mathrm{~K}$ is about $\$ 100$. In comparison a cost of a single broadband ultrasonic transducer can easily be around $\$ 500$ and additional support electronics are required to operate it.

Nevertheless, ultrasonic thermometry offers several advantages over conventional temperature sensors and is unmatched for some of its unique features. Firstly, ultrasound can be used for remote temperature measurements inside an opaque object, for example, the human body. This possibility is important for hyperthermia therapy (tightly controlled overheating of a volume of human tissue containing harmful cells) [10]. Secondly, conventional sensors in fact react not to the temperature of the sensor's environment, but to the temperature of the sensor itself. If the temperature of the environment changes rapidly, conventional sensors require a response time of up to
$10 \mathrm{~s}$ to track the change. In contrast, an ultrasound wave travels over $1 \mathrm{~m}$ in $1 \mathrm{~ms}$ in liquids and solids and thus can track fast changes several orders of magnitude more quickly. An application of ultrasonic thermometry to the measurement of the temperature of firing naval guns was recently developed [11]. Thirdly, an ultrasound wave senses temperature along its complete pathway and thus can replace several conventional sensors that may be difficult to place inside the object of interest, for example, inside a car [12].

Ultrasound propagation in aqueous solutions depends on both the chemical composition and temperature of the solution. Moreover, ignoring temperature-related changes might lead to a poor interpretation of ultrasonic data obtained during process monitoring [13]. In this paper, we discuss two different approaches to ultrasonic temperature measurements of aqueous solutions. The first approach uses continuous wave operation in the through transmission mode with relatively inexpensive narrowband ultrasonic transducers and support electronics. The second approach utilises pulse-echo mode for a wideband ultrasonic transducer and high speed waveform digitiser that are used for high resolution ultrasonic monitoring of liquids. 
The paper is organised as follows. The use of the first approach for measurements in deionised water is discussed in Section 2. Here we present experimental results that complement data reported previously [14]. The second approach uses a dipstick probe that contains a transducer and a reflector that are connected to each other. The clearance area between them is filled when the probe is submerged into the solution of interest. The reflector has a special cavity filled with deionised water [15]. The waveform propagated in this cavity is acquired along with the principal waveform propagated in the clearance area. The propagation delay in the cavity is dependent on the temperature because of temperature dependence of the ultrasound velocity in deionised water [1] and is used for actual temperature measurements after a suitable calibration. Section 3 presents experimental results for measurements in salty solutions of different salinity across a range of temperatures. Section 4 discusses experimental results obtained for temperature measurements in a solution of dynamically varied acidity. The paper is concluded by Section 5 .

\section{Ultrasonic Temperature Measurements Using an Oscillating Sensor}

Hydrophones, which are fabricated with a protective cover, are conventionally used for ultrasonic measurements in water at tens or hundreds of $\mathrm{kHz}$. Because of the relatively high cost and poor off-the-shelf availability of hydrophones, we used low-cost narrowband piezoelectric transducers. Some of them, despite being designed for operation in air, feature a solid case, which offers protection from water and could potentially be used for operation in aqueous solutions. A pair of these transducers was submersed in deionised water for two weeks and no notable changes were found in its operation afterwards.

The use of narrowband transducers makes it difficult to adopt a pulse operation. For continuous wave operation, the phase shift of the wave propagated through the medium under test is to be measured instead of the pulse delay. In order to achieve a good temperature resolution (say, $0.1 \mathrm{~K}$ which is common for conventional thermometers), the resolution of the phase measurements should be rather high. If the propagation distance $l$ is $1 \mathrm{~m}$, the operating frequency $f$ is $40 \mathrm{kHz}$, the ultrasound velocity $c$ is $1500 \mathrm{~m} / \mathrm{s}$ (a close value to that of water), and the change of ultrasound velocity $\Delta c$ with the change of temperature by $0.1 \mathrm{~K}$ is $0.2 \mathrm{~m} / \mathrm{s}$ (a typical value for water), then the change in the phase of the propagated wave equates to:

$$
\begin{aligned}
\Delta \varphi & =\frac{\Delta c}{c} \varphi_{0}=\frac{\Delta c}{c} \times \omega \tau=\frac{\Delta c}{c} \times 360^{\circ} f \frac{l}{c}=360^{\circ} \frac{f l \Delta c}{c^{2}} \\
& =360^{\circ} \frac{40 \times 10^{3} \mathrm{~Hz} \times 1 \mathrm{~m} \times 0.2 \mathrm{~m} / \mathrm{s}}{(1500 \mathrm{~m} / \mathrm{s})^{2}} \approx 1^{\circ},
\end{aligned}
$$

where $\omega$ is the angular frequency and $\tau$ is the propagation delay. This resolution for phase measurement is not easily achievable. For this reason, we used a self-oscillation electronic architecture that is presented in Figure 2.

The circuit produces oscillations at the frequency where the overall gain exceeds one, and the overall phase shift equals to $360^{\circ}$. If the phase shift changes due to a change of temperature, and hence a change in ultrasound velocity, the frequency changes accordingly. Unlike the phase shift, the frequency is easy to measure by counting output pulses during a set time. If this set time is $1 \mathrm{~s}$, the resolution of the frequency measurement equals $1 \mathrm{~Hz}$ which is well below $0.01 \%$ for operation at ultrasonic frequencies.

The experimental arrangement shown in Figure 2 was successfully used for temperature measurements in the range $20 \cdots 35^{\circ} \mathrm{C}$ with a sensitivity of about $280 \mathrm{~Hz} / \mathrm{K}$ at the central frequency of $328 \mathrm{kHz}$ [14]. However, the distance used (30 mm, Figure 2) was not sufficient for many industrial pipes and vessels and the set of experiments reported here used a propagation distance of $190 \mathrm{~mm}$ instead. We first found that the magnitude response of the pair of transducers at this distance (Figure 3) was different to the response obtained at a distance of $30 \mathrm{~mm}$ [14, Figure 4].

Most notably, because of the increased propagation distance and higher attenuation of ultrasound at higher frequencies, the resonances at about $25 \mathrm{kHz}$ became most profound. Therefore, this operating frequency was selected for the experiments reported here.

Further experiments showed that the magnitude response of the closed signal loop was temperature dependant (Figure 4), like it was observed before for higher operating frequency [14]. Additionally, the measured responses exhibited two strong (above $5 \mathrm{~dB}$ ) resonances around $25 \mathrm{kHz}$ with a difference of about $1 \mathrm{kHz}$.

Experiments with the sensor were conducted in a Carbolite-PIF120 thermostat. Vessel 1 (Figure 2) was filled with deionised water, and the water temperature was measured directly by a Digitron 3204 thermometer. The thermostat was heated to $32^{\circ} \mathrm{C}$, and then cooled naturally. The temperature in the vessel and the output frequency of the sensor are presented in Figure 5 for four independent tests.

Although the results for tests 1 and 2, and tests 3 and 4 were consistent, there was a disparity of about $0.5 \mathrm{kHz}$ between the groups. This disparity was attributed to different initial frequencies at which oscillations started for different experiments. The exact reason for observed differences in the initial frequency of oscillations is yet unclear. The sensitivity of the sensor was about $40 \mathrm{~Hz} / \mathrm{K}$ for both groups.

These experimental results show that an ultrasonic oscillating temperature sensor can be used for much higher distances $(190 \mathrm{~mm})$ than had been reported before $(30 \mathrm{~mm})$. The output of the sensor became ambiguous because of the temperature dependence of the magnitude responses and the presence of two resonances instead of one for the transducers used. Both of these reasons are specific to the transducers we used for the experiments and not to the ultrasonic measurement of temperature in general. Therefore, measurements of temperature at higher distances would require uniresonant transducers with ideally no temperature dependence for unambiguous measurements. The major attraction of the oscillating sensor is its low cost. In particular, the retail cost 


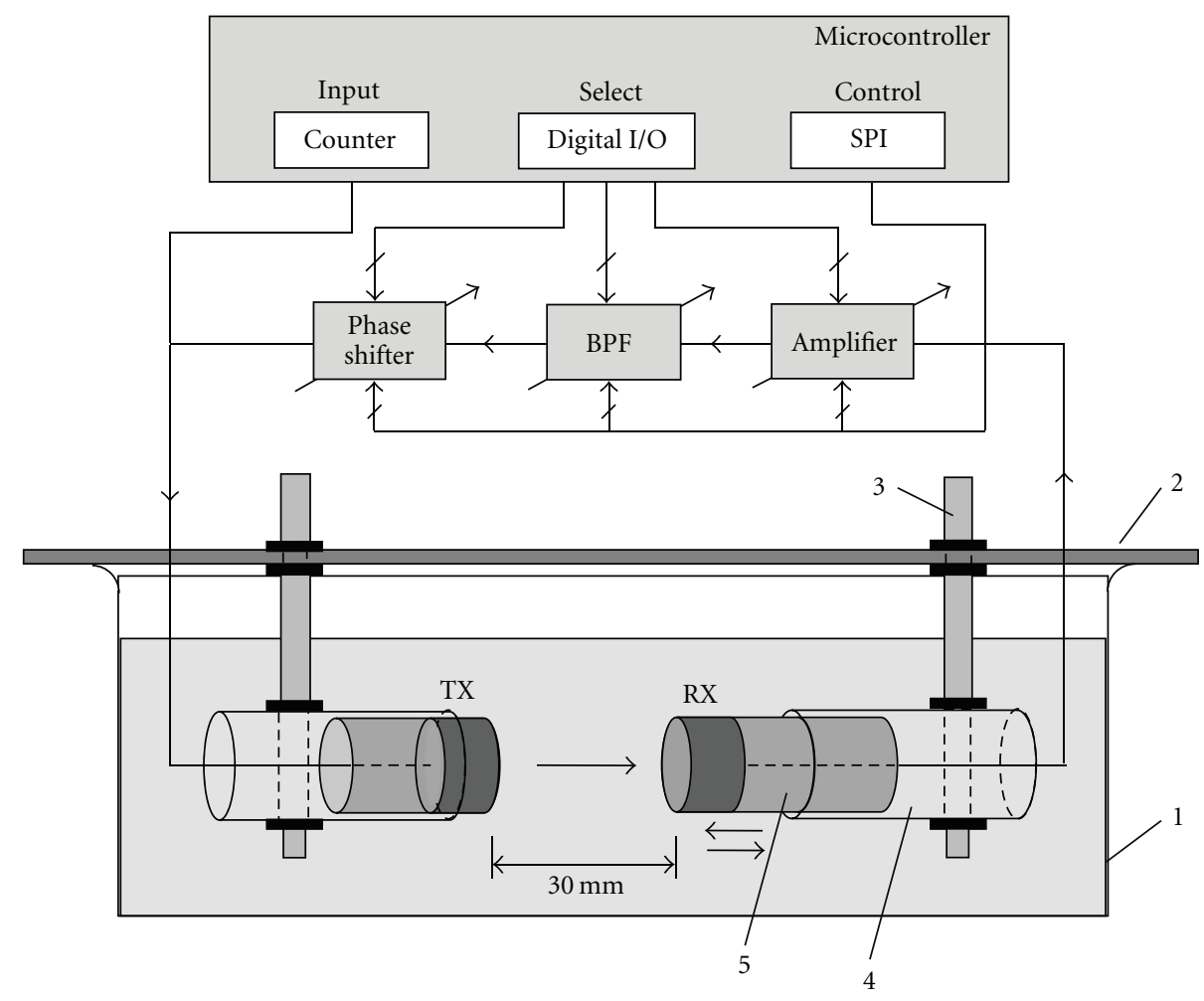

FIgURE 2: Experimental setup for temperature measurements using an oscillating sensor [14].

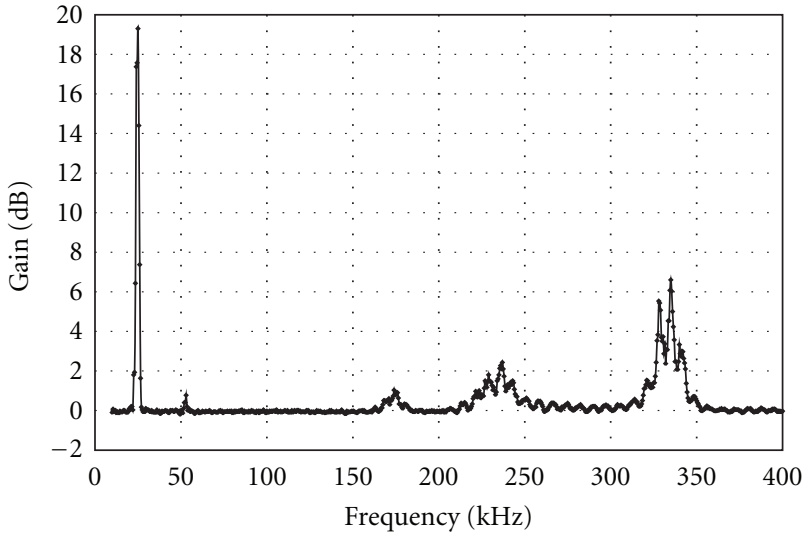

FIGURE 3: Magnitude response of the pair of transducers for the oscillating sensor at a distance of $190 \mathrm{~mm}$.

of the transducers and electronic components used for these experiments was around $\$ 20$.

\section{Ultrasonic Temperature Measurements Using Pulse-Echo Mode for Varied Temperature and Salinity}

Ultrasonic measurements of temperature in the pulse-echo mode were conducted using the experimental setup shown in Figure 6.

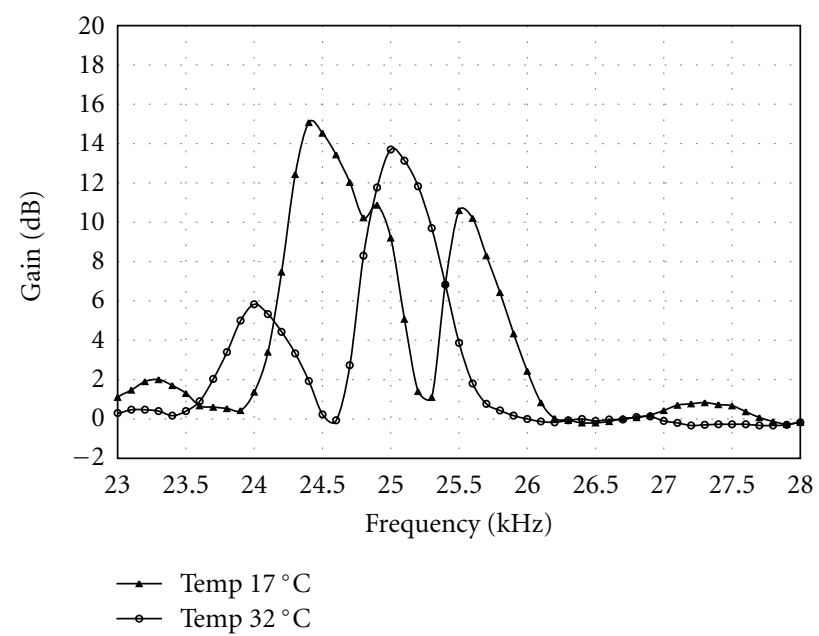

FIgURE 4: Magnitude response of a pair of transducers for an oscillating sensor at a distance of $190 \mathrm{~mm}$ at different temperatures.

The solution under examination was put inside a thermostat Carbolite-PIF120, and the reference temperature measurements were conducted using a Digitron 3204 thermometer (manual recording) and a $\mathrm{pH}-340 \mathrm{i} \mathrm{pH}$-meter for automatic logging of the temperature data. The dipstick probe that housed a $20 \mathrm{MHz}$ ultrasonic transducer featured a specially designed reflector filled with deionised water (Figure 7) [15]. Most of the case of the cavity was made from stainless steel that had a low specific heat capacity but 


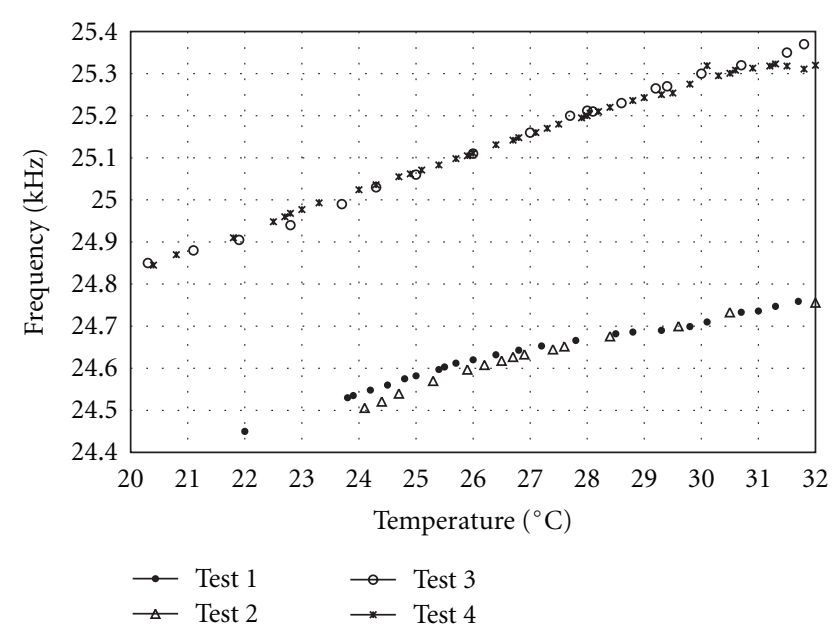

FIGURE 5: Output frequency of an oscillating sensor versus temperature.

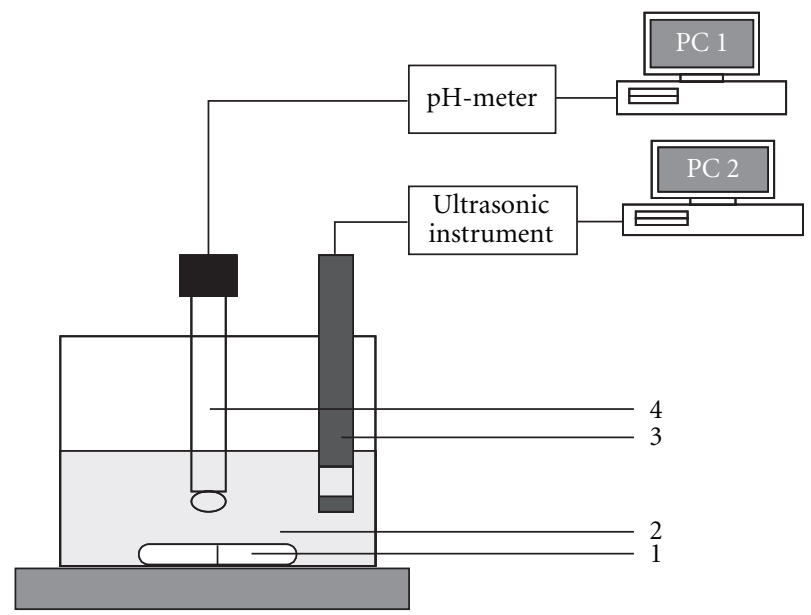

FIGURE 6: Experimental setup for pulse-echo measurements using the dipstick probe (1-magnetic stirrer; 2 -aqueous solution; 3 dipstick probe; $4-\mathrm{pH}$ meter probe).

good thermal conductivity compared to water. Therefore, any changes to the temperature of the aqueous environment were quickly transferred to the water inside the cavity that enabled rapid ultrasonic detection of these changes. The acquired ultrasonic waveforms included the excitation signal, the signal reflected from the front face of the reflector (these were used to calculate the ultrasound propagation delay in the solution), and the signals reflected from the boundaries of the cavity (Figure 7).

The data acquisition system used accurate interleaved sampling with equivalent sampling frequency of $2.7 \mathrm{GHz}$ and employed 1024 averages for every recorded waveform [17]. Measurements itself took about $2 \mathrm{~s}$, and it took another $2 \mathrm{~s}$ to upload the acquired waveform to a hard disk drive of a personal computer. Delay A (in the solution, Figure 7) and delay B (in the cavity, Figure 7) were calculated from the acquired waveforms using a MATLAB script. It implemented time delay estimation based on zero crossings of

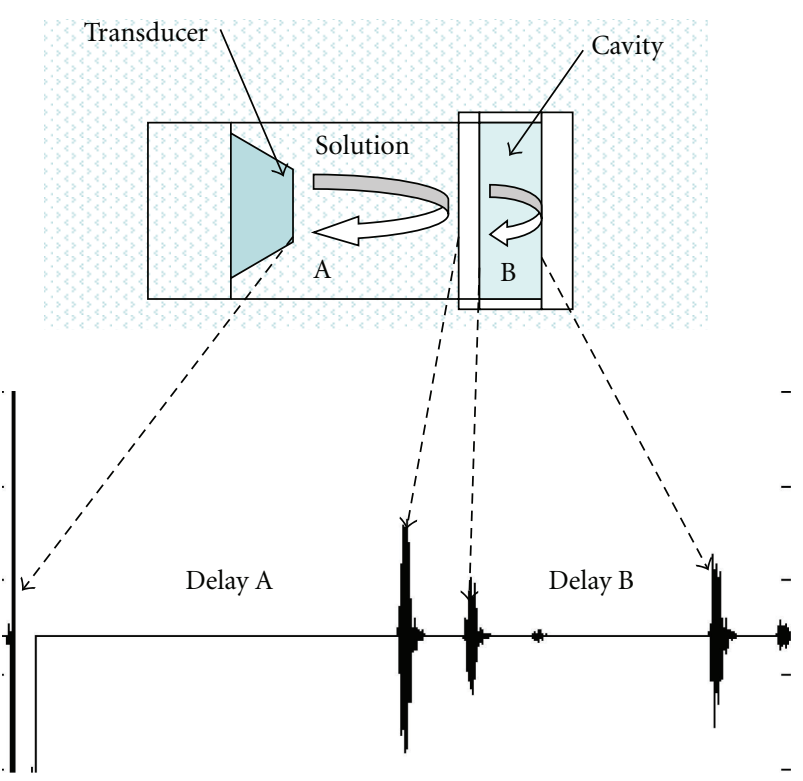

FIGURE 7: Waveforms recorded using a dipstick probe with a water filled cavity.

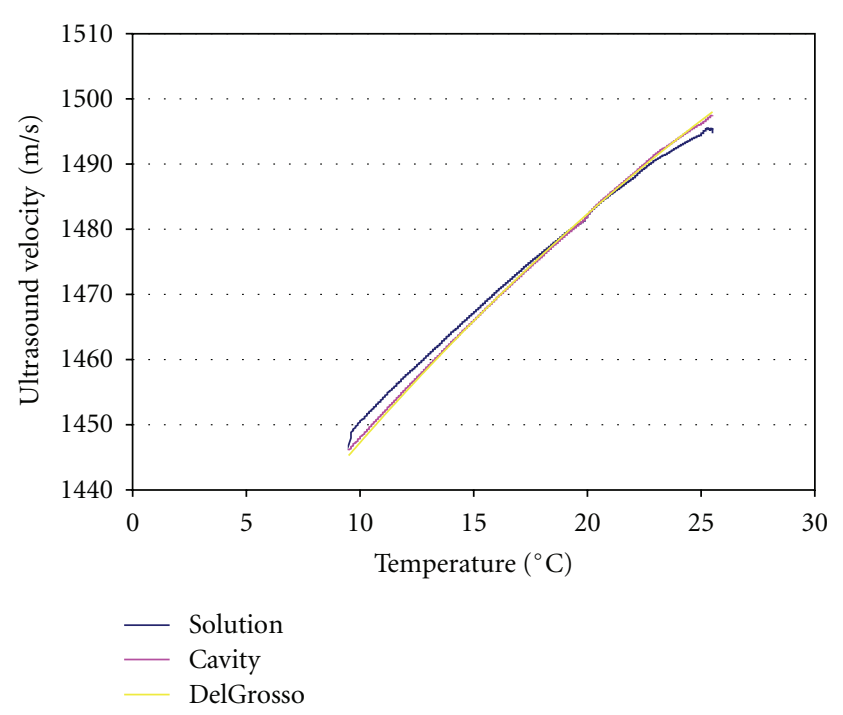

Figure 8: Experimentally measured ultrasound velocities in the solution and in the cavity along with the literature data [16].

the waveform. Linear interpolation between two adjacent samples with different signs was applied to achieve some extra resolution.

The experiments were conducted for deionised water and aqueous solutions of sodium chloride (cooking salt) $\mathrm{NaCl}$ [16] (with concentrations 0.01, 0.05, 0.1, and $0.5 \mathrm{M}$ ) and sodium persulfate $\mathrm{Na}_{2} \mathrm{~S}_{2} \mathrm{O}_{8}$ [18] (with concentrations 0.01 and $0.1 \mathrm{M}$ ). The solutions were heated up in the thermostat, and ultrasonic measurements were taken continuously about every $4 \mathrm{~s}$.

The dipstick probe was first submersed in deionised water for calibration and the expected ultrasound velocities for different temperatures were calculated using Del Grosso 


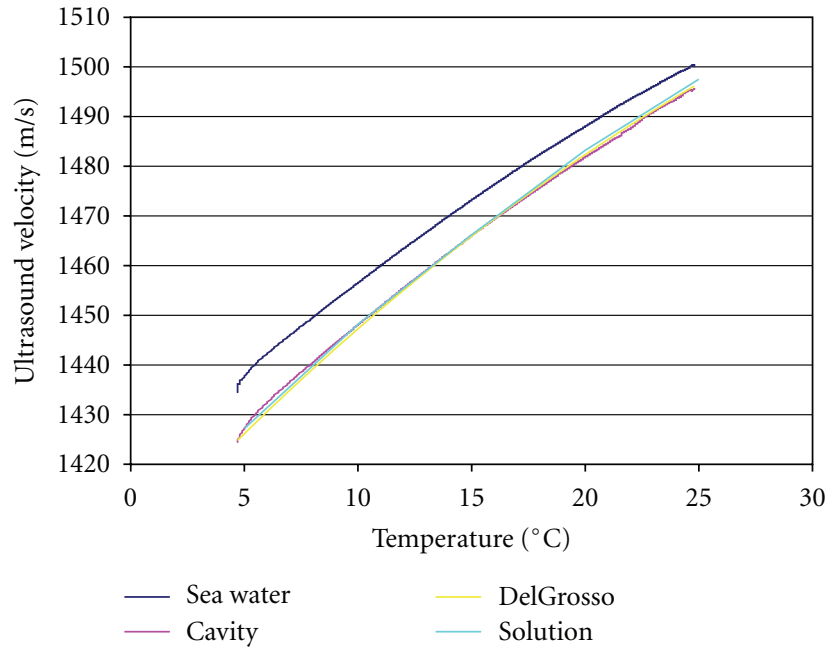

(a)

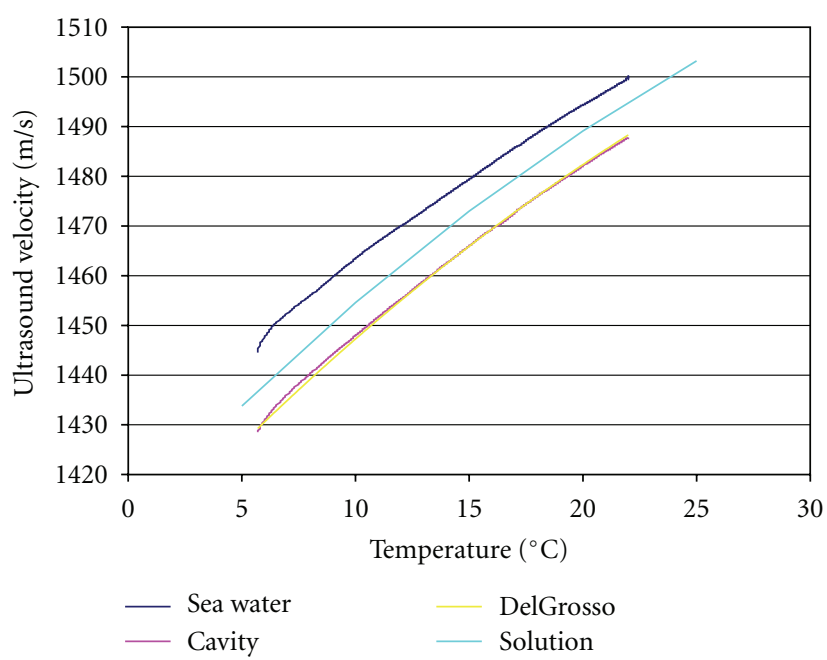

(c)

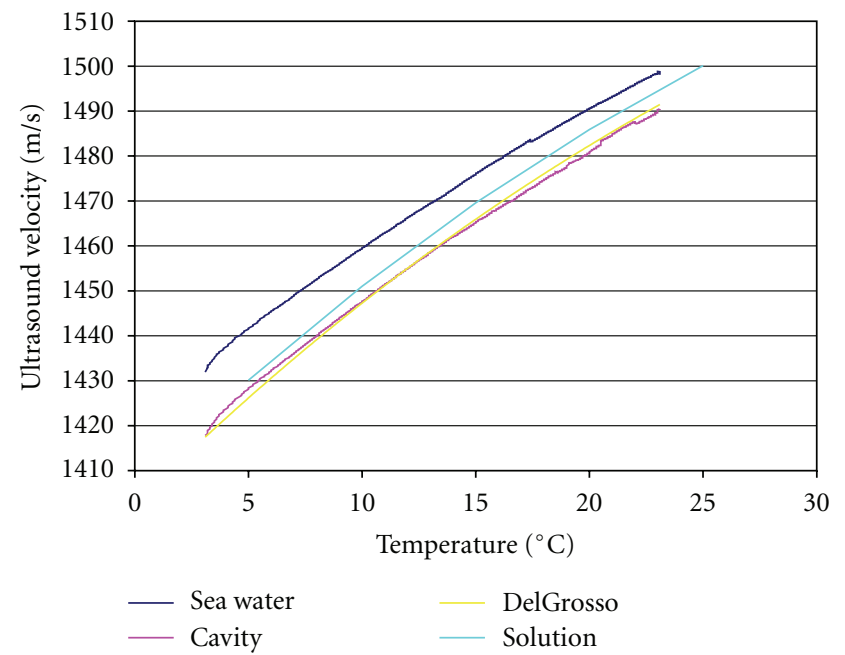

(b)

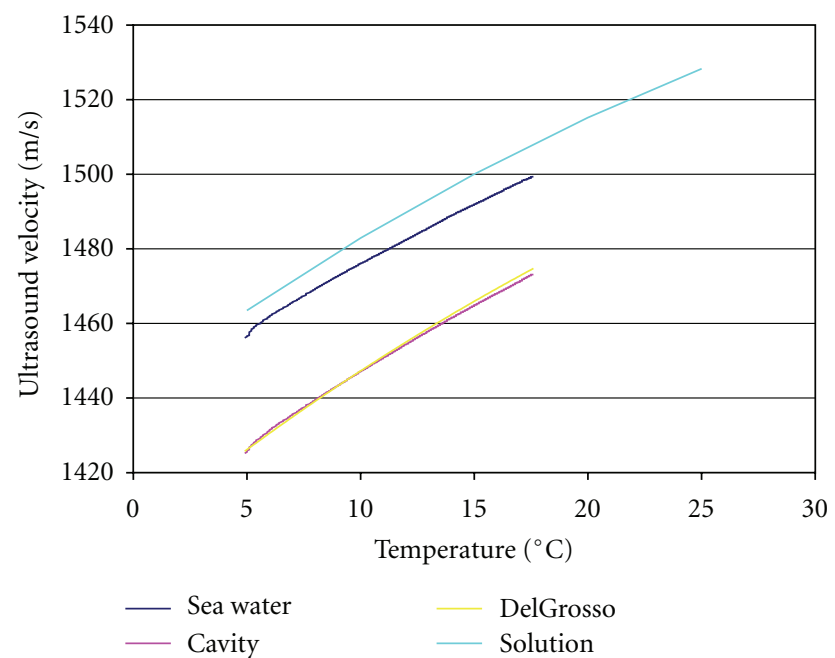

(d)

FIGURE 9: Ultrasound velocities in aqueous solutions of sodium chloride of different concentrations ((a) $0.01 \mathrm{M}$; (b) $0.05 \mathrm{M}$; (c) 0.1 M; (d) $0.5 \mathrm{M}$ ).

and Mader data [19]. The measured width of the cavity was adjusted in order to best fit the predicted ultrasound velocities. The results obtained, presented in Figure 8, show a good agreement between the ultrasound velocities for both the cavity and the solution. The uncertainty of the measurement results caused by the ultrasonic instrumentation alone was estimated based on the frequency domain approach [20], and was found below $0.01 \mathrm{~m} / \mathrm{s}$. Therefore, the thermometer used for the direct temperature measurements could be considered as the principal source of measurement uncertainty.

Unfortunately not much experimental data (and associated empirical models) were published for ultrasound velocity in various aqueous solutions across a range of temperatures. A notable exception was seawater [21], for which an equation for ultrasound velocity was standardised internationally [22] (some similar equations for this purpose were published by other authors. A good discussion on the subject and further references are available online [23]. The list of equations presented there does not seem exhaustive though, e.g., [24]). Although this equation is valid within a limited range of salinities (30-40 ppt with the average salinity of 35 ppt or $0.599 \mathrm{M} \mathrm{[21]),} \mathrm{we} \mathrm{used} \mathrm{it} \mathrm{nevertheless} \mathrm{as} \mathrm{a} \mathrm{reference}$ for our experimental data, (use of this reference, in particular for sodium chloride, is justified by the fact that this salt is the major component of seawater after water itself [21]).

Experimental data showed that the seawater ultrasound velocity model was unsuitable for both chemicals at all the concentrations used (Figures 9 and 10).

Differences in experimental and modelled values of ultrasound velocity were observed even for $0.5 \mathrm{M}$ concentration of sodium chloride (Figure 9(d)), despite the fact that this concentration complies with the range of valid salinities for the model used. 


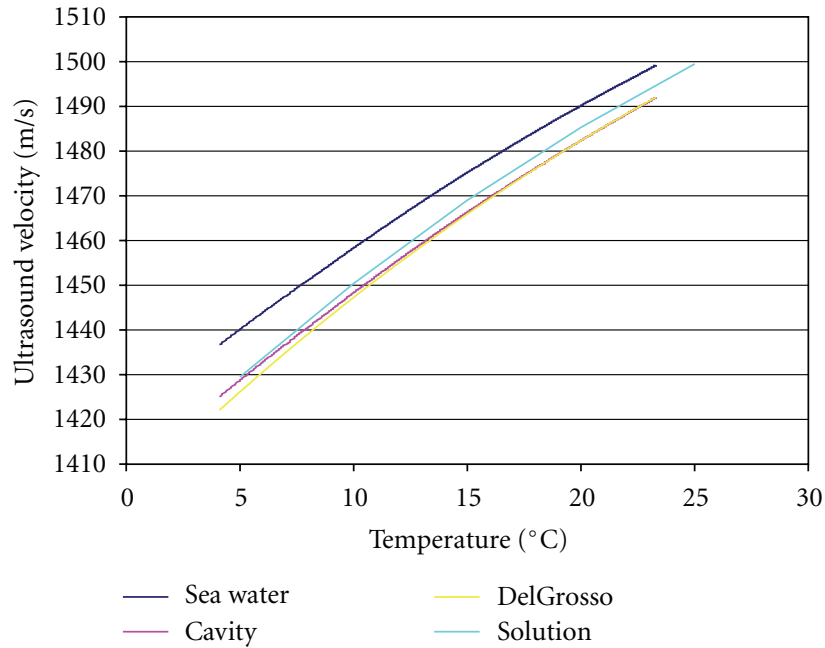

(a)

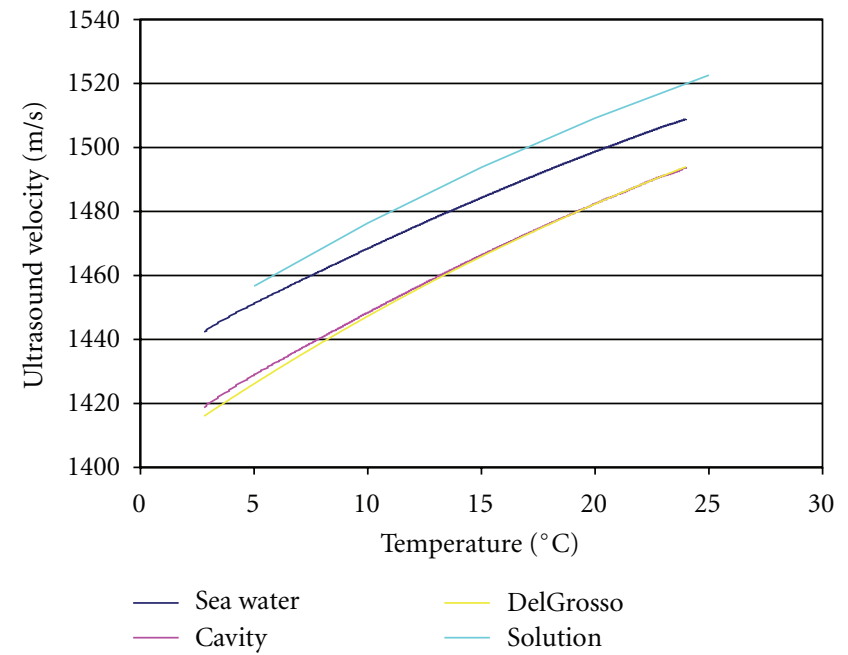

(b)

Figure 10: Experimental ultrasound velocities in aqueous solutions of sodium persulfate ((a) concentration $0.01 \mathrm{M}$; (b) concentration $0.1 \mathrm{M})$.

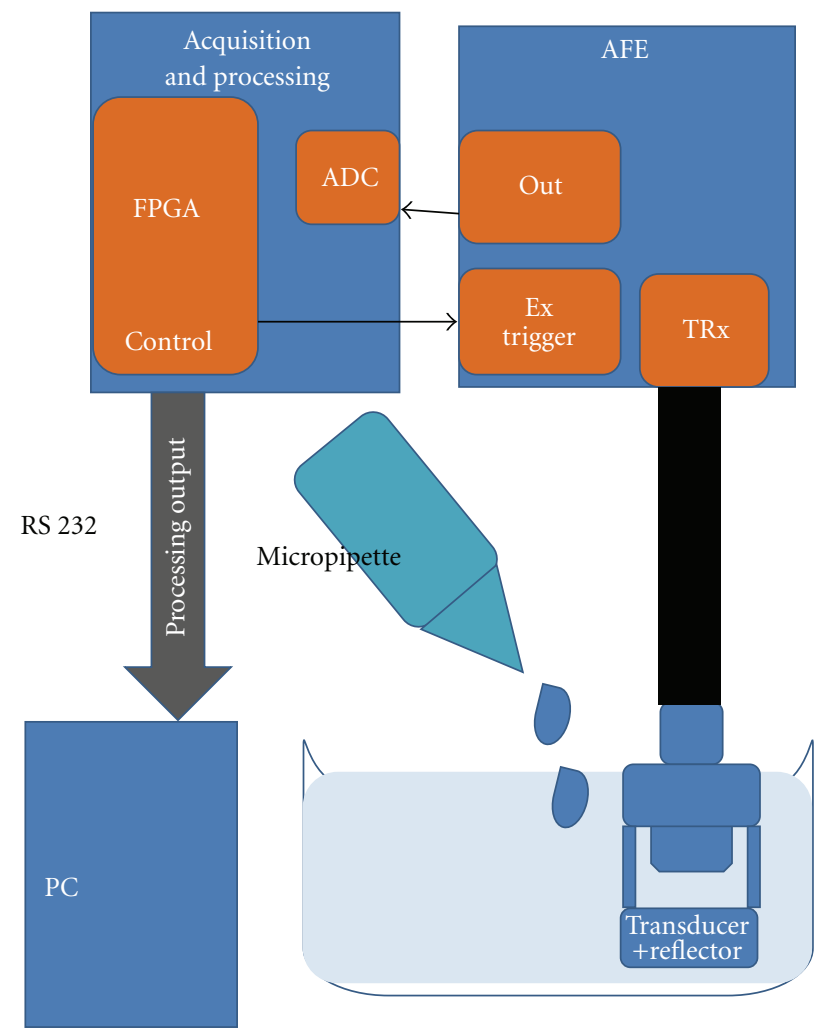

FIGURE 11: Experimental setup for ultrasonic temperature measurements of an aqueous solution with varied concentrations.

Overall, in the set of experiments reported in this section, ultrasound velocity increased along with the increases in temperature and concentration of the dissolved solids as expected. It was found that empirical model developed for calculations of seawater ultrasound velocity was unsuitable for the solutions used in the experiments.

\section{Ultrasonic Temperature Measurements Using Pulse-Echo Mode for Dynamically Varied Acidity}

A dipstick probe with a reflector with a water-filled cavity was used for another set of experiments conducted 


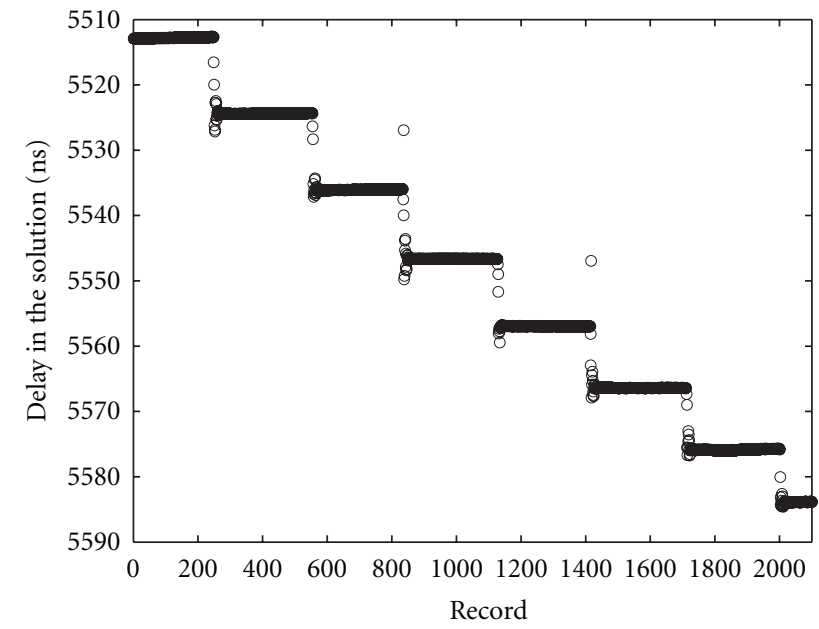

(a)

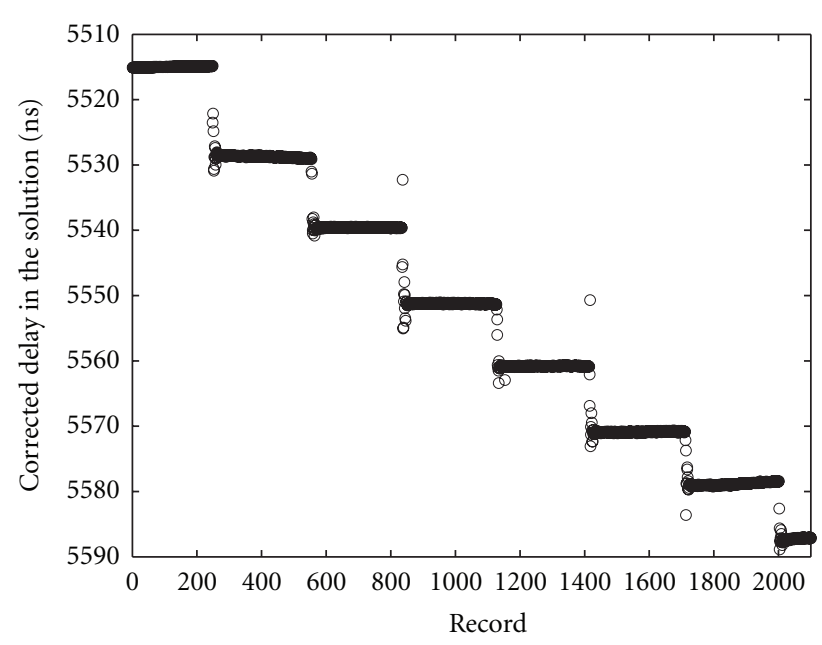

(b)

FIGURE 12: Experimentally obtained propagation delays for varied concentrations of acetic acid in the solution without (a) and with (b) correction.

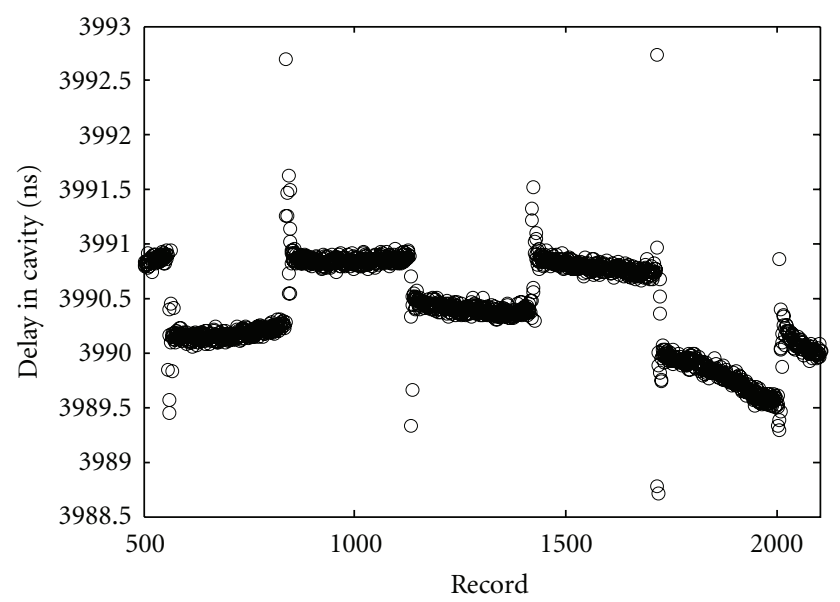

Figure 13: Delays in the cavity during the course of the experiment.

using a recently developed ultrasonic waveform acquisition instrument that consisted of two boards. An off-the-shelf field-programmable gate array (FPGA) board was configured to provide interleaved sampling, signal averaging and embedded processing to determine the propagation delay and communicate it to a personal computer for storing and displaying the delay values. A custom designed board included an analogue-to-digital converter (ADC) and an analogue front end (AFE) that were connected to the transducer and the FPGA board (Figure 11). An embedded MicroBlase processor performed evaluation of the propagation delay itself, without the need of uploading the waveform to a personal computer. This made it possible to take 5 measurements of propagation delay every second at the equivalent sampling frequency of $1 \mathrm{GHz}$ with 512 averages. Embedded processing enabled a substantial reduction of the minimal time interval between successive measurements compared to the design outlined in the previous section, (that design required around $2 \mathrm{~s}$ for waveform uploading alone, and $4 \mathrm{~s}$ per a single measurement overall).

The experiments were conducted by adding $1 \mathrm{~mL}$ of chemical (99\% acetic acid $\left.\mathrm{CH}_{3} \mathrm{COOH}[25]\right)$ to $160 \mathrm{~mL}$ of deionised water every 1 minute. The delay in the cavity was used for ultrasonic measurements of the temperature while a Digitron 3204 thermometer was used as the reference for direct temperature measurement.

The experimentally determined propagation delays in the solution are presented in Figure 12(a).

During the experiment the reference thermometer displayed a constant temperature of $23.8^{\circ} \mathrm{C}$, whilst the delay in the cavity showed notable variation (Figure 13).

We believe that, being carefully calibrated, the temperature readings obtained ultrasonically were more accurate. We used the delays from Figure 13 to adjust the experimentally recorded data to the same temperature of $25^{\circ} \mathrm{C}$ in the following way:

$$
d_{\text {corr }}=d_{\exp }(t) \times \frac{d_{\mathrm{dw}}\left(25^{\circ} \mathrm{C}\right)}{d_{\mathrm{dw}}\left(t_{\exp }\right)},
$$

where $d_{\text {corr }}$ is the corrected propagation delays at $25^{\circ} \mathrm{C}$; $d_{\text {exp }}$-experimental propagation delays in the aqueous solution of interest determined at temperature $t ; d_{\mathrm{dw}}-$ propagation delay in deionised water. This correction assumes that the ultrasound velocity in the solution behaves with temperature in the same way as in deionised water. Although (2) presents an assumption only, it seems reasonable to us for adjustment of the experimental values to the same temperature that was required for meaningful comparison and plotting. The corrected propagation delays in the solution are presented in Figure 12(b).

The corrected values for the propagation delays were used to find a best fit straight line for the ultrasound velocity versus the concentration (Figure 14). 


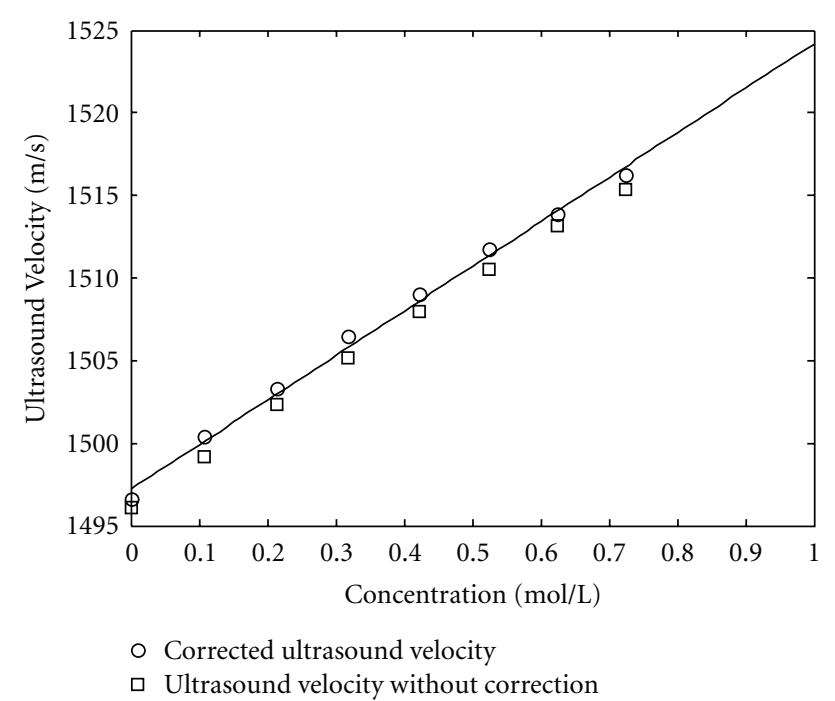

FIGURE 14: Ultrasound velocity versus concentration for acetic acid solutions (measured at around $23.8^{\circ} \mathrm{C}$ and corrected to $25^{\circ} \mathrm{C}$ ).

The results show that, without the correction, the best fit line at zero concentration of the acetic acid would be biased compared to the ultrasound velocity in the deionised water $\left(1496.7 \mathrm{~m} / \mathrm{s}\right.$ for $\left.25^{\circ} \mathrm{C}\right)$. That is why the applied correction seems essential to us.

The experiments reported in this section showed that ultrasonic temperature measurements with the dipstick probe were able to detect and quantify very small changes of temperature when a chemical was injected to the solution. These temperature changes were not detectable by a conventional thermometer with a resolution of $0.1 \mathrm{~K}$.

Experimental values of ultrasound velocity were corrected in order to refer the measurements to the same temperature. For this correction, we assumed that the temperature dependence of the ultrasound velocity in the aqueous solution was broadly the same as that of the deionised water. The ultrasound velocity in the solution increased along with the increases in acidity, and showed nearly linear dependence on the acid concentration.

\section{Summary and Conclusions}

In this paper, two different approaches were used for measurement of temperature in the aqueous solutions.

Oscillating temperature sensor operated at the frequency of about $25 \mathrm{kHz}$ in the continuous wave mode, and showed sensitivity of about $40 \mathrm{~Hz} / \mathrm{K}$ at the distance of $190 \mathrm{~mm}$. The advantage of this sensor is low cost of the transducer and support electronics (around \$20), although the transducers need to be bettered in order to provide unambiguous readings in a wide temperature range. These desirable improvements to the transducers include lesser dependence of their magnitude responses on the temperature, and elimination of unwanted resonances by design. This sensor seems most suitable for permanent installation in a liquid tank or a process vessel of interest.
Ultrasonic temperature sensing at the frequency of $20 \mathrm{MHz}$ was performed using a dipstick probe that can be immersed in various liquid tanks or process vessels with an open top. This sensor seems more suitable for inspection of different places in a liquid tank, where the probe can be moved manually or using an actuator. It demonstrated very high resolution for either temperature or chemical composition when the other variable was kept constant. Ultrasound velocity measurements alone cannot discriminate between these two possible causes of the ultrasound velocity change. Therefore, ultrasonic evaluation of temperature for aqueous solutions with evolving composition additionally requires monitoring of this composition and preliminary calibration. The sensitivity of the ultrasound velocity to changes in temperature and salinity can be approximately estimated from the published data (see [22]) as $5.0(\mathrm{~m} / \mathrm{s}) / \mathrm{K}$ and $82(\mathrm{~m} / \mathrm{s}) / \mathrm{M}$, respectively.

Both sensors seem to require a calibration in a thermostat before use. This is because the temperature measurements with resolution of $0.1 \mathrm{~K}$ require measurement of ultrasound velocity with accuracy better than $0.01 \%$. As this accuracy is directly dependent on the accuracy of mechanical dimensions that determine the ultrasound propagation length, for example, the width of the dipstick cavity, the mechanical parts have to be manufactured expensively with very small tolerances otherwise.

The support electronics for the sensors needed to provide high measurement resolution (better than $0.01 \%$ as stated above). For the oscillating sensor, this resolution can be traded for the measurement time, the higher measurement time allowing more accurate frequency measurements. As for the dipstick sensor, the resolution was determined by the accuracy of the propagation delay measurements. This time was measured by using high equivalent sampling frequencies in excess of $1 \mathrm{GHz}$ for echo waveforms, and applying linear interpolation for finding a relevant zero crossing.

The measurement results showed that in the used aqueous solutions, the ultrasound velocity was notably different from the velocity in seawater for the same salinity at the same temperature. Therefore, ultrasonic measurements can be potentially used for discrimination among close chemical substances provided that they are kept at the same temperature.

\section{Acknowledgments}

A. N. Kalashnikov and V. Ivchenko thank the EPSRC (UK) for their support for developments of novel electronic architectures for ultrasonic instruments. S. Alzebda thanks Said Foundation and Leche Trust for their support of his PhD study. A. Afaneh thanks the University of Nottingham for their support of his Ph.D. study.

\section{References}

[1] G. W. C. Kaye and T. H. Laby, Tables of Physical and Chemical Constants, Longman, London, UK, 1986.

[2] A. M. Mayer, "On an acoustic pyrometer," Philosophical Magazine, vol. 45, pp. 18-22, 1873. 
[3] A. A. Fathimani and J. F. W. Bell, "A new resonant thermometer for nuclear reactor applications," Journal of Physics E, vol. 11, no. 6, pp. 588-596, 1978.

[4] S. F. Green, "An acoustic technique for rapid temperature distribution measurements," Journal of the Acoustical Society of America, vol. 77, no. 2, pp. 759-763, 1985.

[5] L. C. Lynnworth, "Industrial applications of ultrasound. A review. II. Measurements, tests, and process control using low intensity ultrasound," IEEE Transactions on Sonics and Ultrasonics, vol. 54-22, no. 2, pp. 71-101, 1975.

[6] "Thermocouple," http://en.wikipedia.org/wiki/Thermocouple.

[7] "Resistance thermometer," http://en.wikipedia.org/wiki/Resistance_thermometer.

[8] "Thermistor," http://en.wikipedia.org/wiki/Thermistor.

[9] "Silicon bandgap temperature sensor," http://en.wikipedia org/.wiki/Silicon_bandgap_temperature_sensor.

[10] R. M. Arthur, W. L. Straube, J. W. Trobaugh, and E. G. Moros, "Non-invasive estimation of hyperthermia temperatures with ultrasound," International Journal of Hyperthermia, vol. 21, no. 6, pp. 589-600, 2005.

[11] D. E. Yuhas, M. J. Mutton, J. R. Remiasz, and C. L. Vorrez, "Ultrasonic measurements of bore temperature in large caliber guns," Review of Progress in Quantitative NDE, July 2008, http://imsysinc.com/downloads/QNDE\%20NETS.pdf.

[12] T. L. Liao, W. Y. Tsai, and C. F. Huang, "A new ultrasonic temperature measurement system for air conditioners in automobiles," Measurement Science and Technology, vol. 15, no. 2, pp. 413-419, 2004.

[13] A. N. Kalashnikov, K. L. Shafran, V. G. Ivchenko, R. E. Challis, and C. C. Perry, "In situ ultrasonic monitoring of aluminum ion hydrolysis in aqueous solutions: instrumentation, techniques, and comparisons to $\mathrm{pH}$-metry," IEEE Transactions on Instrumentation and Measurement, vol. 56, no. 4, pp. 13291339, 2007.

[14] S. Alzebda and A. N. Kalashnikov, "Ultrasonic sensing of temperature of liquids using inexpensive narrowband piezoelectric transducers," IEEE Transactions on Ultrasonics, Ferroelectrics and Frequency Control, vol. 57, pp. 2704-2711, 2011.

[15] A. N. Kalashnikov, V. Ivchenko, R. E. Challis, and A. K. Holmes, "Compensation for temperatre variation in ultrasonic chemical process onitoring," in Proceedings of the IEEE Ultrasonics Symposium, pp. 1151-1154, September 2005.

[16] "Sodium chloride," http://resource.npl.co.uk/acoustics/techguides/soundseawater/.

[17] A. N. Kalashnikov, V. G. Ivchenko, R. E. Challis, and B. R. Hayes-Gill, "High-accuracy data acquisition architectures for ultrasonic imaging," IEEE Transactions on Ultrasonics, Ferroelectrics, and Frequency Control, vol. 54, no. 8, pp. 15961605, 2007.

[18] "Sodium persulfate," http://en.wikipedia.org/wiki/Sodium_persulfate.

[19] V. A. Del Grosso and C. W. Mader, "Speed of sound in pure water," Journal of the Acoustical Society of America, vol. 52, no. 5, pp. 1442-1446, 1972.

[20] A. N. Kalashnikov and R. E. Challis, "Errors and uncertainties in the measurement of ultrasonic wave attenuation and phase velocity," IEEE Transactions on Ultrasonics, Ferroelectrics, and Frequency Control, vol. 52, no. 10, pp. 1754-1768, 2005.

[21] "Seawater," http://en.wikipedia.org/wiki/Seawater.

[22] G. S. K. Wong and S. M. Zhu, "Speed of sound in seawater as a function of salinity, temperature, and pressure," Journal of the Acoustical Society of America, vol. 97, no. 3, pp. 1732-1736, 1995.
[23] "Speed of sound in sea-water," http://resource.npl.co.uk/ acoustics/techguides/soundseawater/.

[24] J. R. Lovett, "Merged sea-water sound speed equations," Journal of the Acoustical Society of America, vol. 63, no. 6, pp. 1713-1718, 1978.

[25] "Acetic acid," http://en.wikipedia.org/wiki/Acetic acid. Publications of the present authors are available for anonymous download on $\mathrm{ftp}: / / 128.243 .76 .5 /$ PUBLIC DISK/ak1/index .htm. 

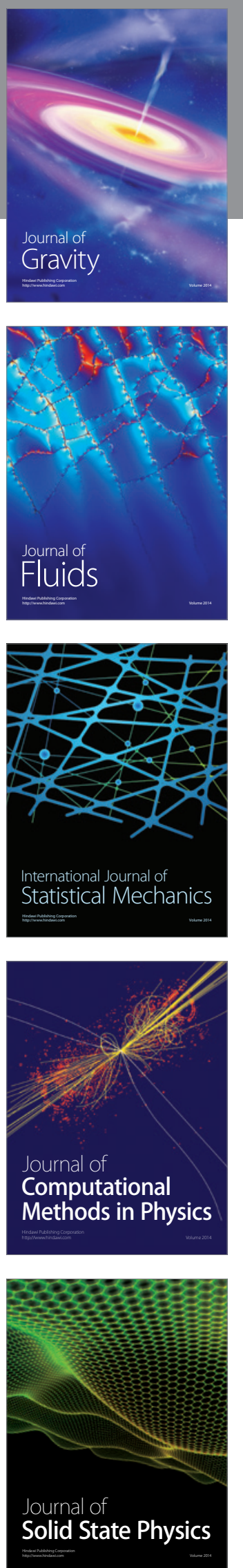

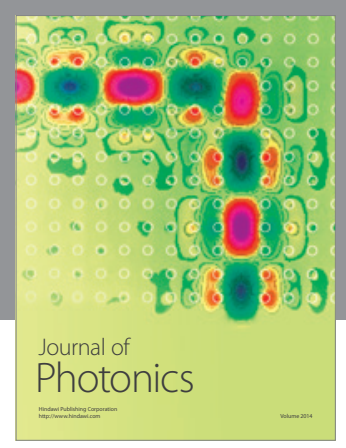

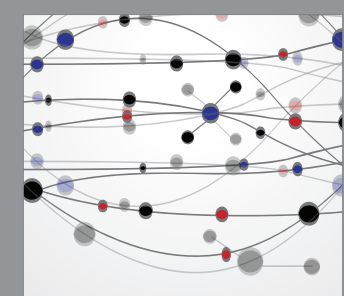

The Scientific World Journal
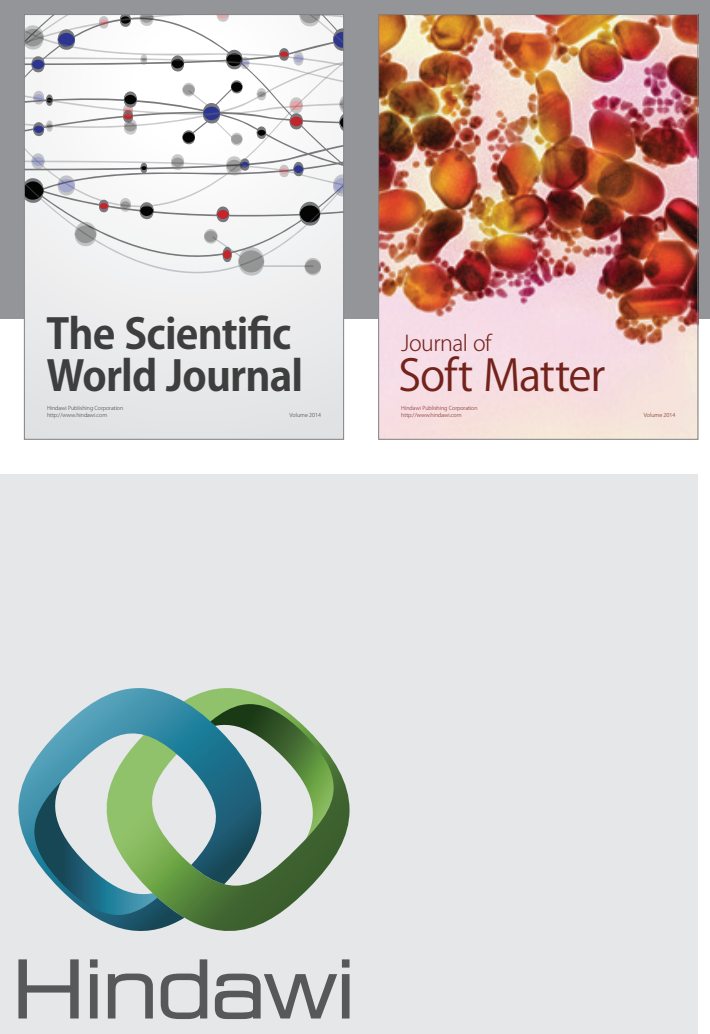

Submit your manuscripts at

http://www.hindawi.com
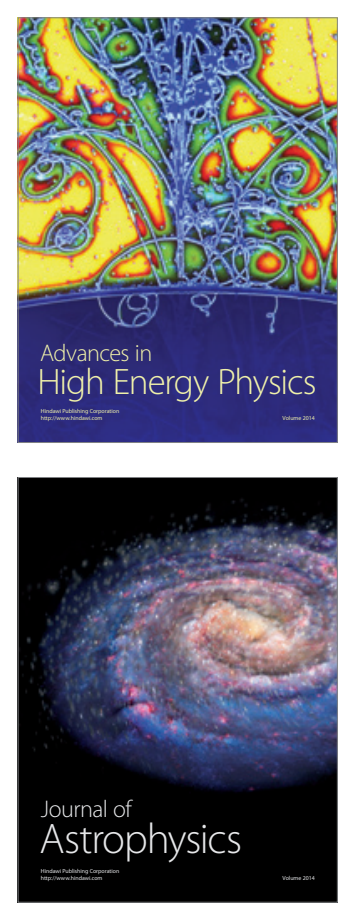
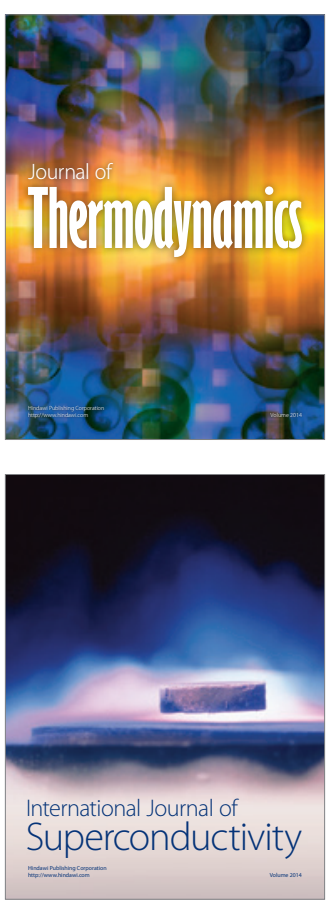
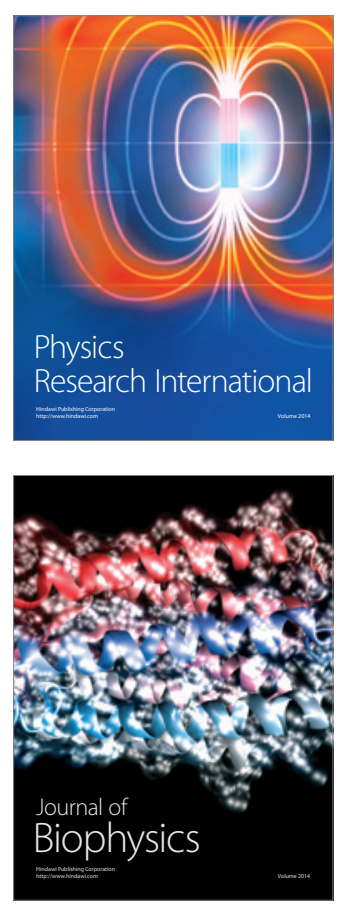
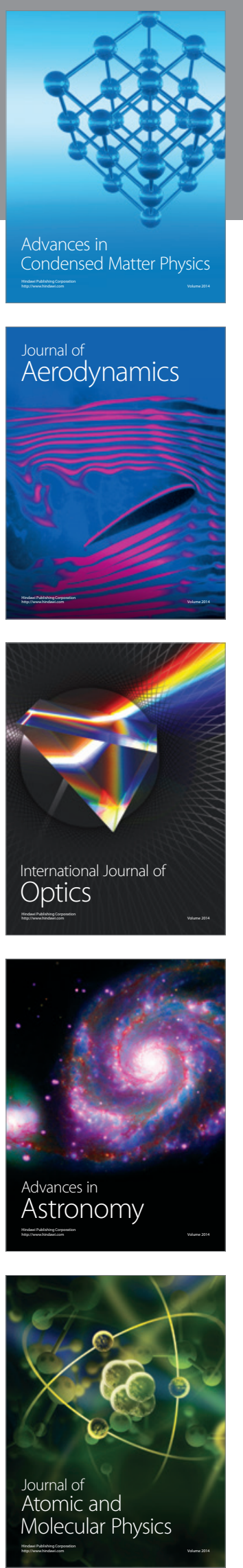